\title{
Adicción a las Tecnologías: Adolescencia, familias y Trabajo Social. Revisión teórica del fenómeno
}

\section{Technology addiction: \\ Adolescence, families and Social Work. Theoretical review of the phenomenon}

\author{
CARLOTA ROCA BELIJAR \\ Graduada en Trabajo Social por la Universidad de Murcia. Máster en Género e lgualdad.carlota.roca@um.es
}

Recibido: 04/09/2019

Aceptado: 31/10/2019

\begin{abstract}
Resumen: Las Tecnologías de la Información y Comunicación (en adelante, TIC), se encuentran totalmente difundidas en nuestra sociedad actual, tanto es así que ya no es extraño ver a cualquier persona llevando y/o utilizando un móvil o un ordenador con observable asiduidad. Por otra parte, tampoco es menos cierto el hecho de que las mismas se constituyen como una de las herramientas de más utilidad hoy en día, muchas son las ventajas que presentan, permiten desde facilitar la comunicación o relaciones hasta comprar sin salir de casa, por ejemplo. Sin embargo, ¿todo en ellas es positivo?, en los últimos tiempos se está constatando el nacimiento de un nuevo fenómeno que afecta especialmente a adolescentes, y es que, son cada vez más frecuentes los casos en los que éstos/as desarrollan patrones de conducta nocivos o adictivos en relación con el uso de las $\mathrm{TIC}^{1}$. Este hecho, puede llegar a tener graves consecuencias, por ejemplo, en la vida familiar de los/as más jóvenes debido a que es precisamente la familia uno de los contextos más próximo o cercano. Así, a lo largo de este artículo se realizará un recorrido teórico por alguna de las cuestiones que giran en torno a una adicción a las TIC, así como a propuestas de abordaje desde perspectiva del Trabajo Social.
\end{abstract}

Palabras clave: Tecnologías de la Información y Comunicación, adicción, familia, adolescencia, Trabajo Social

\begin{abstract}
Information and Communication Technologies (ICT), are fully disseminated in our current society, so much so that it is no longer strange to see anyone carrying and/or using a mobile or a computer with observable assiduity. On the other hand, it is not less true the fact that they are constituted as one of the most useful tools today, there are many advantages that they present, they allow from facilitating communication or relationships to buying without leaving home, for example. However, is everything in them positive?, in recent times, the birth of a new phenomenon that especially affects adolescents is being verified, and that is, the cases in which they develop patterns of harmful or addictive behavior in relation to the use of ICT. This fact, can have serious consequences, for example, in the family life of the youngest as the family is precisely one of the closest or closest contexts. Throughout
\end{abstract}

1 El concepto de adicción a las TIC en este artículo hará referencia al establecimiento de patrones nocivos hacia cualquier tipo de dispositivo digital (móvil, Tablet...), actividad lúdica (videojuegos, apuestas online...) o aplicaciones (twitter, whatsapp, Instagram...) derivadas de dichas TIC. 
this article there will be a theoretical tour of some of the issues that revolve around an addiction to ICT, as well as proposals for approach from the perspective of Social Work.

Keywords: Information and communication technologies, addiction, family, adolescence, Social Work.

\section{INTRODUCCIÓN. ACERCAMIENTO AL CONCEPTO DE ADICCIÓN A LAS TIC}

La implantación de las TIC de forma masiva en casi todos los ámbitos de la vida y en todas las franjas es ya un hecho comprobable, aunque si bien cabe apuntar que tal y como se expondrá más adelante, la última encuesta realizada por el Instituto Nacional de Estadística (2018) acerca de "Equipamiento y uso de tecnologías de información y comunicación en los hogares" y más concretamente el apartado "Uso de TIC por los menores", apunta de forma clarificadora que los sectores de la población que más utilizan las TIC son los correspondientes a los/as adolescentes.

Hoy en día verdaderamente es extraño encontrar a un/a adolescente que no utilice una Tablet, o a un/a adolescente que no tenga y que no utilice un móvil, un ordenador o algún otro dispositivo, y no es que el uso de las TIC en sí sea algo negativo ni que haya que suprimirlas por completo cuando estamos hablando de sectores de la población como adolescentes pero... ¿qué ocurre cuando los/as más jóvenes sobrepasan la fina línea que existe entre lo que podría entenderse como un entretenimiento y lo que se puede considerar una verdadera adicción? Actualmente la sociedad se enfrenta a, tal y como refiere el Psicólogo especializado en Psicología Clínica de la Salud, Pedro Martínez, "Un nuevo perfil de paciente. Personas jóvenes que se muestran tan dependientes como en el caso de los adictos a cualquier droga" (Citado en Téllez, 2018, párr.7).

Las TIC y sus distintos dispositivos están totalmente incorporados a la vida cotidiana de la ciudadanía de la sociedad occidental del Siglo XXI. Si nos remontamos a su aparición, a lo largo de los años se ha podido constatar que han supuesto un gran avance en todos los sentidos, es indudable el hecho de que las mismas mejoran o facilitan la vida de las personas al permitirnos agilizar el trabajo, acceder a información de todo tipo de forma realmente sencilla, estar en contacto con otras personas por muy lejos que éstas estén e incluso ya hasta realizar acciones tan diarias o comunes como hacer la compra o pedir una cita médica o de otro tipo sin salir de casa. De esta forma, parece que no hay nada que no sea realmente positivo en ellas, pero no del todo pues si nos centramos en los/as más jóvenes, ya es algo extraño no encontrar a adolescentes de cada vez más temprana edad portando consigo un móvil, Tablet o consola a cualquier hora del día o utilizando todos estos aparatos tecnológicos como prácticamente única forma de entretenimiento y comunicación, pudiendo desembocar toda esta rutina en una posible creación de patrones de uso nocivos. En base a esto, ahondando en el término adicción relacionado con las TIC y a su vez con los/as adolescentes, es sustancial conocer a qué nos referimos exactamente cuando hablamos de este fenómeno. Es importante decir que no todos/as los/as jóvenes que manejan y utilizan las TIC de forma habitual presentan una adicción.

Algunas de las definiciones más relevantes son las siguientes que aparecen ordenadas de forma cronológica desde los primeros estudios conocidos sobre este tema hasta más actuales:

Según Echeburúa (1994):

La adicción a Internet puede considerarse una adicción específicamente psicológica con características comunes a otros tipos de adicciones: pérdida de control, aparición de síndrome de abstinencia, fuerte dependencia psicológica, interferencia en la vida cotidiana y pérdida de interés por otras actividades (Citado en Muñoz y Ramírez, 2016, p. 47).

Según Young (1996):

La adicción a Internet es un deterioro en el control de su uso que se manifiesta como un conjunto de síntomas cognitivos, conductuales y fisiológicos. Es decir, la persona "netdependiente" realiza un uso excesivo de Internet lo que le genera una distorsión de sus objetivos personales, familiares o profesionales. (Citado en Luengo, 2004, p. 25).

Según Griffiths (1997), lo que existe es lo que se denomina como "Adicciones tecnológicas" y las define como: "Adicciones no químicas que involucran la interacción hombre-máquina" (Citado en Luengo, 2004, p. 25).

Según Pratarelli y Cols (1999): "Fenómeno o desorden putativo que depende tanto del medio como del mensaje" (Citado en Álvarez y Rodríguez, 2012, p.90). 
Según la Organización Mundial de la Salud (OMS) (2015): "Uso compulsivo, repetitivo y prolongado con incapacidad para controlar o interrumpir su consumo y consecuencias sobre la salud, la vida social, familiar, escolar o laboral" (Citado en Atienza, 2017, párr.3)

Según Domínguez (2017): "Actividades diversas, a menudo placenteras no asociadas a la ingestión de sustancias psicoactivas" (párr.6).

Cuando un/a adolescente presenta un comportamiento abusivo llegando a adictivo con respecto a las TIC, se nos plantean numerosas preguntas como por ejemplo, ¿de qué manera pueden verse afectados algunos de los diferentes ámbitos de la vida de los/as más jóvenes como por ejemplo el familiar?, ¿cómo pueden ser conscientes las familias de que sus hijos e hijas tienen realmente un problema relacionado con la utilización abusiva de las TIC?, ¿en qué sentido puede ayudar la disciplina del Trabajo Social en estos temas?

El objetivo primordial que persigue este artículo es el de dar respuesta a las preguntas anteriores, así como proponer desde un punto de vista personal, ideas de prevención y tratamiento sobre la perspectiva del Trabajo Social. El método utilizado ha sido la realización de una exhaustiva revisión bibliográfica de documentos procedentes de bases de datos (Dialnet o Google Académico), informes oficiales de instituciones públicas y privadas (Instituto Nacional de Estadística, Centro de Investigaciones Sociológicas, Ministerio de Sanidad o clínica privada Cita especializada en adicciones), revistas científicas (Revista Katharsis, Trabajo Social Hoy, Adicciones o Revista Profesional Española de Terapia Cognitivo Conductual) y periódicos (El País o BBC Mundo) que versan sobre el tema a tratar en este artículo. Además, también se reflejan investigaciones o estudios de carácter tanto nacional como internacional que han sido seleccionados para ser expuestos por sus resultados realmente novedosos, claros y representativos.

El artículo se encontrará dividido en tres primeras partes de carácter contextualizador (conceptos, pruebas diagnósticas y datos de actualidad) y dos segundas partes más orientadas a las consecuencias y abordaje de la problemática (secuelas familiares y propuestas de intervención)

\section{CATALOGACIÓN DE LA ADICCIÓN Y PRINCIPALES TEST O PRUEBAS DE EVALUACIÓN}

Algo especialmente relevante en este artículo es señalar cómo este tipo de adicciones de las que se viene hablando han sido y son enfocadas desde la perspectiva del mayor de los referentes en clasificaciones de trastornos mentales, el denominado como Manual Diagnóstico y Estadístico de los Trastornos Mentales de la Asociación Americana de Psiquiatría, conocido comúnmente con las siglas DSM (Asociación Americana de Psiquiatría (2016) Manual diagnóstico y estadístico de trastornos mentales ( $5^{\mathrm{a}}$ ed., Texto rev.)).

En la anterior versión de este Manual Diagnóstico, el DSM-IV, no se incluía ningún tipo de clasificación que con carácter único hiciera referencia a las denominadas como "Adicciones comportamentales o sin sustancia" (grupo del que forman parte las TIC al ser un tipo de adicción que no precisa de la ingesta de ningún tipo de sustancia), simplemente éstas se encontraban incluidas de manera más bien general en el epígrafe "Trastorno del Control de los Impulsos". En años posteriores, la nueva versión, el conocido como DSM-V cuya publicación y última modificación en España datan de los años 2014 y 2016 respectivamente, avanza y ya incluye una categoría denominada como "Trastornos no relacionados a sustancias", sin embargo, es preciso aclarar que esta nueva versión no hace referencia a que dicha categoría sea equivalente a decir "Adicciones comportamentales" ya que en la misma simplemente se incluyen los Trastornos por Juegos de Apuestas en sentido totalmente desvinculado y no referencial a las TIC.

Siguiendo en la línea del DSM-V, es importante señalar que aunque la adicción a las TIC no haya sido incluida en esta versión del Manual Diagnóstico, el mismo sí que deja una puerta abierta para una posible introducción aunque sea parcial en el futuro ya que en su Sección III sobre "Condiciones para más estudios en el futuro", cita a los denominados "Trastornos por Juegos de Internet" a los cuales viene a definir de manera resumida, como aquellos a los que el/la usuario/a les llega a dedicar hasta 8 o 10 horas diarias, siendo éstos más frecuentes en adolescentes. En base a esto, es muy posible que esta cuestión anterior y otras relacionadas con la adicción a las TIC, sean incluidas próximamente en este famoso Manual Diagnóstico. (Pedrero, Rodríguez y Ruiz, 2012).

A continuación, también es importante exponer los principales mecanismos que se han ido estableciendo y que se utilizan todavía en la actualidad como referentes para intentar conocer o determinar si existe o no una adicción a las TIC de cara a facilitar la detección de este tipo de adicciones sobre todo a la familia y entorno más cercano de la persona víctima. De esta forma, a destacar:

Young (1998) establece una serie de señales de alarma que se pueden constituir como unos indicadores dirigidos a ayudar a las familias a identificar una adicción a las TIC en sus adolescentes (Echeburúa y Corral, 2010). (Tabla I) 


\section{Tabla I. Señales de alarma sobre una posible adicción a las TIC Según Young}

\section{Señales de alarma}

1. Privarse de sueño para estar conectado a la red, a la que se dedica unos tiempos de conexión anormalmente altos.

2. Descuidar otras actividades importantes, como el contacto con la familia, las relaciones sociales, el estudio o el cuidado de la salud.

3. Recibir quejas en relación con el uso de la red de alguien cercano, como los progenitores o los/as hermanos/as

4. Pensar en la red constantemente, incluso cuando no se está conectado a ella y sentirse irritado excesivamente cuando la conexión falla o resulta muy lenta.

5. Intentar limitar el tiempo de conexión, pero sin conseguirlo, y perder la noción del tiempo.

6. Mentir sobre el tiempo real que se está conectado o jugando a un videojuego.

7. Aislarse socialmente, mostrarse irritable y bajar el rendimiento en los estudios.

8. Sentir una euforia y activación anómalas cuando se está delante del ordenador.

Fuente: Echeburúa y Corral (2010)

\section{Tabla II. CRiterios diagnósticos para la detección de la adicción a las TIC de Young}

\section{Criterios Diagnósticos}

1. Sentimiento de preocupación por Internet (piensa acerca de anteriores o futuras actividades en línea)

2. Siente la necesidad de aumentar la cantidad de tiempo usando Internet para alcanzar satisfacción

3. Ha realizado esfuerzos repetidos sin éxito para controlar, disminuir o detener el uso de Internet

4. Se ha sentido inquieto/a, malhumorado/a, deprimido/a o irritable en sus intentos de parar o detener el uso de Internet

5. Ha estado más tiempo del que pretendía en Internet

6. Ha estado en riesgo de perder alguna relación importante, un trabajo o una oportunidad de educación debido a Internet

7. Ha mentido a miembros de su familia, al terapeuta o a otros para ocultar la importante relación con Internet

8. Usa Internet como camino para escapar de problemas o para aliviar un disgusto

Fuente: Sigüenza y Fernández (2016)

\section{TABLA III. Test de Adicción a LAS TIC de ECheburúa}

Test de adicción a las TIC

1. ¿Te sientes preocupado/a con Internet (pensamientos acerca de la última conexión o anticipas la próxima conexión)?

2. ¿Sientes la necesidad de incrementar la cantidad de tiempo de uso de Internet para lograr la satisfacción?

3. ¿Ha hecho repetidamente esfuerzos infructuosos para controlar, reducir o detener el uso de Internet?

4. ¿Se ha sentido inquieto/a, malhumorado/a, deprimido/a o irritable cuando ha intentado reducir o detener el uso de Internet?

5. ¿Se queda más tiempo conectado del que inicialmente había pensado?

6. ¿Ha perdido o puesto en peligro alguna relación significativa, trabajo, oportunidad educativa o profesional debido al uso de Internet? 7. ¿Ha mentido a los miembros de su familia, terapeuta u otros para ocultar su grado de implicación con Internet?

8. ¿Usa Internet como un medio para evadirse de los problemas o de aliviar su estado de ánimo disfórico?

Fuente: Luengo (2004)

De nuevo Young (1999) establece una serie de criterios diagnósticos para determinar la existencia o no de una adicción a las TIC tomando como base el presentar al menos 5 de estos 8 criterios siguientes (Sigüenza y Fernández, 2016). (Tabla II)

Echeburúa (1999), elabora un test de adicción constituido por un total de 8 ítems de respuesta SI/NO. Así, las puntuaciones de entre 4-8 son indicativas de un cierto riesgo de padecer una dependencia y las puntuaciones de entre 7-8 son indicativas de un problema de dependencia ya establecido (Luengo, 2004). (Tabla III)

\section{DATOS RELEVANTES EN RELACIÓN CON LOS ADOLESCENTES Y LAS TIC}

En primer lugar en este apartado, es preciso citar un suceso al que se alude en la noticia "La OMS reconoce el tras- 


\begin{tabular}{|c|c|c|c|}
\hline \multicolumn{4}{|c|}{ TABLA IV. Porcentaje de Menores usuarios de TIC por sexo y edad. Año 2018. Encuesta INE } \\
\hline & Uso de ordenador (\%) & Uso de Internet (\%) & Disposición de móvil (\%) \\
\hline Total & 91,3 & 92,8 & 69,8 \\
\hline \multicolumn{4}{|l|}{ Sexo } \\
\hline Hombres & 90,4 & 92,5 & 68,2 \\
\hline Mujeres & 92,2 & 93,2 & 71,6 \\
\hline \multicolumn{4}{|l|}{ Edad } \\
\hline 10 & 85,7 & 85,6 & 26,2 \\
\hline 11 & 88,5 & 86,3 & 41,1 \\
\hline 12 & 92,5 & 92,4 & 75,1 \\
\hline 13 & 92,5 & 96,2 & 86,2 \\
\hline 14 & 93,0 & 97,0 & 91,2 \\
\hline 15 & 95,0 & 98,7 & 94,8 \\
\hline
\end{tabular}

Fuente: INE (2018)

torno por videojuegos como problema mental", ya que tal y como indica Salas (2017, párr.1) en la misma, esto que se cita en el titular se recogerá en la próxima edición de la Clasificación Internacional de Enfermedades (en adelante, CIE-11), que además no se actualiza desde el año 1990. Este hecho como se puede deducir, es debido al creciente número de casos que día a día continúan apareciendo sobre todo en la población adolescente. Dicho esto, es importante reflejar con el ánimo de facilitar su entendimiento, no crear falsas alarmas en las familias y aunque no está del todo estructurada la inclusión de este tipo de adicción comentada en la CIE-11, el borrador de la misma indica que la OMS se basa claramente en al menos tres criterios para poder diagnosticar esta patología de tal dificultad y envergadura:

1. Falta de control sobre el juego: Este síntoma implica que la persona es incapaz de controlar su conducta hacia el videojuego y no puede poner límite al tiempo que invierte en esa actividad ni a las veces que lo hace durante el día o la semana.

2. Aumento de la prioridad que se le da al juego: Se convierte en algo por encima de otros intereses vitales y rutinas del día a día.

3. Continuación o escalada del juego, a pesar de las consecuencias negativas: No importa el daño que esté haciendo a su salud o a su situación vital, el afectado continúa jugando o lo hace cada vez más. (BBC Mundo, 2018).

En segundo lugar, es importante hacer referencia a la última encuesta del Instituto Nacional de Estadística (en adelante, INE) sobre "Equipamiento y uso de tecnologías de información y comunicación en los hogares" publicada el 7 de Noviembre de 2018 y basada en una recogida de información acerca del uso de las TIC (Internet, telefonía móvil u ordenador) por la población española. En la misma, lo que nos interesa resaltar es el apartado referido al "Uso de TIC por los menores" ya que es ahí donde se ve reflejada una amplia visión de lo que pasa en nuestra sociedad. (Tabla IV)

Como se observa en la encuesta, hay más niñas y adolescentes mujeres que utilizan y se relacionan con las TIC en comparación con los adolescentes hombres aunque la diferencia tampoco sea muy acusada. Además, también destacar que a partir de los 12 años, el número de chicas y chicos que se convierten en usuarios/as de las TIC se incrementa de forma exponencial, lo que a edades tan tempranas se constituye como datos como mínimo llamativos. (INE, 2018).

A la hora de indagar en la existencia de una posible adicción a las TIC en jóvenes, es importante estudiar el tiempo que los/as mismos/as dedican a lo largo de su día a los distintos dispositivos y entretenimiento que ofrecen dichas TIC. Así, es preciso hacer referencia a los resultados obtenidos en una reciente investigación realizada por García, Piqueras y Marzo (2017) en la cual se analiza el uso problemático de internet, móvil y videojuegos en adolescentes alicantinos teniendo como muestra a 319 jóvenes de entre 12 y 18 años.

En referencia al uso problemático de Internet: (Tabla V) 


\section{Tabla V. Porcentajes de frecuencia de uso de Internet, SEgún GRUPOS de edad y SEXo}

\begin{tabular}{lcccc} 
& A diario $(\%)$ & $2-3$ veces por semana (\%) & Una vez por semana (\%) & De vez en cuando (\%) \\
\hline $12-13$ años & 66,3 & 22,5 & 2,5 & 8,8 \\
\hline $14-16$ años & 92,6 & 6,6 & 0,0 & 0,7 \\
\hline 17 -18 años & 98,0 & 2,0 & 0,0 & 0,0 \\
\hline Chicos & 83,9 & 10,9 & 1,5 & 3,6 \\
\hline Chicas & 87,6 & 10,1 & 0,0 & 2,3 \\
\hline Total & 85,7 & 10,5 & 0,8 & 3,0 \\
\hline
\end{tabular}

Fuente: García, Piqueras y Marzo (2017)

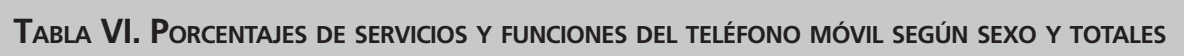

\begin{tabular}{|c|c|c|c|}
\hline & Chicos (\%) & Chicas (\%) & Total (\%) \\
\hline Llamadas & 37,3 & 55,8 & 46,3 \\
\hline Videollamadas & 9,1 & 6,2 & 7,7 \\
\hline SMS & 3,9 & 8,3 & 6,1 \\
\hline Correo electrónico & 22,2 & 18,6 & 20,4 \\
\hline WhatsApp & 94,2 & 97,9 & 96 \\
\hline Redes sociales & 72,5 & 83,4 & 77,7 \\
\hline Páginas web & 64,8 & 59 & 61,9 \\
\hline Cámara de fotos & 60,1 & 90,4 & 74,8 \\
\hline Grabar y ver vídeos & 46,4 & 56,2 & 51,2 \\
\hline Reproducir música & 85,0 & 92,4 & 88,6 \\
\hline Agenda & 13,7 & 42,7 & 27,9 \\
\hline Juegos & 55,5 & 31,8 & 43,9 \\
\hline Otras aplicaciones (Apps) & 72,6 & 63,2 & 68,0 \\
\hline
\end{tabular}

Fuente: García, Piqueras y Marzo (2017)

\section{TABLA VII. FreCuencia de uso de VIDEOJUEGos SEGÚN EDAD Y SEXo}

\begin{tabular}{lcccc} 
& A diario $(\%)$ & $2-3$ veces por semana (\%) & Una vez por semana (\%) & De vez en cuando (\%) \\
\hline $12-13$ años & 9,5 & 33,8 & 14,9 & 41,9 \\
\hline $14-16$ años & 16,8 & 41,6 & 9,7 & 31,9 \\
\hline $17-18$ años & 16,2 & 21,6 & 10,8 & 51,4 \\
\hline Chicos & 18,2 & 50,8 & 11,4 & 19,7 \\
\hline Chicas & 8,7 & 14,1 & 12,0 & 65,2 \\
\hline Total & 14,3 & 35,7 & 11,6 & 38,4 \\
\hline
\end{tabular}

Fuente: García, Piqueras y Marzo (2017)

Sobre uso problemático del móvil: (Tabla VI)

Acerca del uso problemático de videojuegos: (Tabla VII)

Sobre los resultados de estas 3 últimas Tablas, lo que se puede observar es que el uso de las diversas oportunidades que ofrecen las TIC reflejadas, se realiza de forma diaria y asidua por parte de los/as adolescentes objeto de estudio y que, al igual que ocurría con los resultados de la Tabla IV, son más las chicas usuarias que los chicos cuyo porcentaje de estos últimos es menor en la mayoría de categorías descritas. Así, lo que se muestra es la preocupante y masiva implantación y normalización de las TIC en la vida diaria de los/as jóvenes que, siguiendo las orientaciones de Garrote (2013), parece estar desembocando en el favorecimiento de una educación que fomenta la conducta rápida dejando de permitir la reflexión y el pensamiento crítico en los/as mismos/as. Esto, se puede ver reflejado en la decisión tomada por el Ministerio 
de Sanidad de incluir por primera vez las adicciones a las nuevas tecnologías (en referencia a cualquier tipo de dispositivo o entretenimiento relacionado con las mismas) en el Plan Nacional de Adicciones de 2018, destacando la preocupación que existe sobre todo por los/as adolescentes a los que se señala como el mayor grupo de riesgo. Además, Josep María Fàbregas (2018), director de la clínica privada Cita en Dosrius (Barcelona) que trabaja en temas relacionados con las adicciones, incide en que los/as adolescentes no presentan la madurez suficiente como para sobrellevar de manera responsable todo lo que las TIC y en concreto en este caso, los móviles, pueden ofrecerles. Por ello, aboga y secunda la idea de que por ley se restrinja el uso de móviles en menores de 16 años pues según su propio ejemplo, "La ley no permite conducir a un menor de 14 años, aunque sepa llevar un coche, porque consideramos que no tiene suficiente madurez para hacerlo. Podemos plantear lo mismo con el móvil" (Citado en Segura, 2018, párr.7)

\section{CONSECUENCIAS FAMILIARES DERIVADAS DEL USO ADICTIVO DE LAS TIC POR PARTE DE ADOLESCENTES}

El uso de las TIC por parte de los más jóvenes puede entrañar todo tipo de consecuencias negativas sobre los/ as mismos/as cuando éstas se utilizan de forma abusiva y/o adictiva. Dichas consecuencias tienen un amplio espectro de aparición, ya que no sólo afectan a un aspecto de la vida de una persona, sin embargo, siguiendo la línea del tema protagonista de este artículo, se centrará la atención en las consecuencias que ocurren en el ámbito familiar.

La familia constituye el ambiente donde los/as adolescentes pasan gran parte de su tiempo, en ese ámbito del que los/ as mismos/as extraen e interiorizan todo tipo de conductas y aprendizajes. En los últimos años, ha dejado de ser un misterio el hecho de que las dinámicas familiares han cambiado, la rutina estresante, el día a día cargado de responsabilidades laborales, sociales... al que muchos padres y madres se encuentran sometidos hacen que la proximidad con sus hijos/as se vea ciertamente mermada.

La irrupción de las TIC en el ámbito familiar, han transformado por completo las formas de comunicación y hábitos familiares, si bien se erigen como un mecanismo útil para que los progenitores puedan conocer en todo momento el paradero de sus hijos/as, no es menos cierta la cuestión de que la utilización abusiva de las TIC, en este caso por parte de los/as adolescentes, origina importantes consecuencias en el ámbito familiar. Un ejemplo o prueba de esto que se comenta se puede ver reflejado en la investigación llevada a cabo por Ángel y
Alzate (2015) con una muestra de un total de 114 adolescentes de entre 10-17 años y sus progenitores procedentes de los programas académicos de educación superior de la Fundación Universitaria Católica y de los programas de educación básica y media del Cibercolegio UCN (Chile). Dicha investigación se centra en conocer cómo han cambiado las dinámicas familiares y cuáles son las consecuencias que la población encuestada observa en alusión exclusivamente a la relación entre adolescentes/progenitores/TIC. De esta forma, las principales conclusiones derivadas del estudio son las siguientes:

a) Reducción del tiempo y de las actividades compartidas en familia

b) Dificultades en la comunicación

c) Distanciamiento y/o aislamiento familiar: los/as adolescentes adictos/as a las TIC dedican gran parte del tiempo de su día a día a la utilización de las mismas, lo que conduce a la reducción considerable de los espacios temporales, de comunicación y/o de ocio que se comparten con la familia

d) Pérdida de autoridad

e) Incumplimiento de las normas: los/as adolescentes pueden perder la percepción que tienen de sus progenitores como figura de autoridad debido a la poca relación que mantienen con ellos a causa de su adicción a las TIC, lo que conlleva que sea especialmente dificultoso el que los/as mismos/as acaten algún tipo de norma

f) Sentimiento de invasión de la privacidad: Muchos/as adolescentes pueden sentir que el hecho de que sus padres indaguen o se preocupen por el uso que le dan a las TIC, preguntando por ejemplo, con quién hablan o qué tipos de fotos suben a las redes es una intromisión en su privacidad

g) Conflictos familiares: Esta consecuencia vendría a ser la traducción o el resultado de todas las anteriores.

Otra de las cuestiones oportunas a resaltar en este apartado sería hacer alusión al último barómetro hasta ahora realizado y publicado por el Centro de Investigaciones Sociológicas (en adelante, CIS) que recoge concretamente en una pregunta, con su respuesta en término porcentuales, la percepción de la población encuestada (sólo personas que han utilizado redes sociales virtuales en los último seis meses y tienen hijos/as de 12 o más años) acerca de la irrupción de las TIC en la vida familiar. De esta forma:

Pregunta 41: "¿Considera Ud. que en los últimos años el uso de las nuevas tecnologías (Internet, teléfono móvil...), ha 


\begin{tabular}{|c|c|c|c|c|c|c|}
\hline & $\begin{array}{c}\text { Ha aumentado } \\
(\%)\end{array}$ & $\begin{array}{c}\text { Ha disminuido } \\
(\%)\end{array}$ & $\begin{array}{c}\text { Permanece igual } \\
(\%)\end{array}$ & N.S. (\%) & N.C. (\%) & $(\mathrm{N})(\%)$ \\
\hline La comunicación entre padres e hijos & 9,9 & 68,3 & 16,5 & 4,9 & 0,2 & $(2.467)$ \\
\hline El conflicto familiar & 52,4 & 7,6 & 29,0 & 10,6 & 0,4 & $(2.467)$ \\
\hline Las relaciones con los/as abuelos/as & 9,9 & 40,4 & 39,7 & 9,5 & 0,4 & $(2.467)$ \\
\hline La protección de la intimidad & 4,3 & 81,4 & 8,1 & 5,9 & 0,3 & $(2.467)$ \\
\hline La comunicación en la pareja & 7,9 & 52,0 & 30,0 & 9,5 & 0,5 & $(2.467)$ \\
\hline El hacer más cosas juntos & 6,5 & 53,0 & 32,0 & 8,0 & 0,4 & $(2.467)$ \\
\hline
\end{tabular}

Fuente: Elaboración propia a partir de CIS (2016)

hecho que en la vida familiar haya aumentado o disminuido...?" (Tabla VIII)

Como se ha podido observar a través de los diferentes resultados de las investigaciones reflejadas, las consecuencias familiares derivadas del impacto de la aparición de las TIC tienen unas dimensiones verdaderamente considerables ya que pueden verse afectadas áreas como la comunicación familiar y muy alteradas las dinámicas y funciones propias de la familia pues tal y como ya indicaban Echeburúa y Corral (2010): "El tiempo invertidos por los/as adolescentes en la red, puede ir en detrimento de la comunicación y afectividad en el ámbito familiar próximo" (Citado en Ángel y Alzate, 2015, p.4).

\section{PROPUESTAS DE ACTUACIÓN DESDE EL TRABAJO SOCIAL}

El Trabajo Social ha abarcado históricamente la atención a la juventud, familias y adicciones, no obstante, en el ámbito de la adicción específica a las TIC, se configura como una materia que necesita de una mayor profundización. Sin embargo, el Trabajo Social puede ofrecer numerosas opciones de tratamiento, acompañamiento, ayuda, asistencia... no sólo a los/as adolescentes con problemas de adicción a las TIC sino también a sus familias por su carácter holístico y asistencial. Por ello, algunas propuestas de intervención podrían ser:

1. Capacitación de padres y madres: Con normalidad las actuaciones en este ámbito de la adicción a las TIC en jóvenes se centran en dicha población, sin embargo, una propuesta de intervención diferente, sería la configuración de una serie de sesiones adscritas a una secuencia de talleres destinados tanto a los/as padres y madres que se encuentran en una situación en la que sus hijos/as presentan una adicción a las TIC, como también, de un modo preventivo, a todos/as aque- llos/as padres y madres que estén interesados/as en asistir sin que necesariamente sus hijos/as presenten un comportamiento adictivo en relación a las TIC. En base a esto, los talleres se encontrarían encaminados a que los progenitores adquieran habilidades para que por ellos/as mismos/as sean capaces de reconducir la situación de adicción a las TIC por parte de sus hijos/ as mediante estrategias basadas en el establecimiento de una correcta:

\section{- Escucha activa}

- Establecimiento de normas y límites saludables y adecuados: Un/a profesional del Trabajo Social con formación en intervención con el sistema familiar puede trabajar con los progenitores en la adquisición de habilidades para la imposición de normas y límites saludables a sus hijos/as con la mayor firmeza posible pero teniendo en cuenta que lo que se pretende es la interiorización de manera educativa de que hay ciertas "líneas" que no se pueden transgredir.

- "Reeducación" de padres y madres de cara a que interioricen que el mayor ejemplo son ellos/as y que no hagan un mal uso de las TIC delante de sus hijos/as cuando están pidiendo a su misma vez que los/as jóvenes sean responsables.

2. Espacios de encuentro: La organización de espacios de encuentro dirigidos a adolescentes que quieren superar su adicción a las TIC, o a padres y madres que se encuentran pasando por esta situación con sus hijos/as.

3. Familiarización de adolescentes con el uso adecuado y responsable de las TIC mediante la organización de un mayor número de taller dinámicos o de seminarios organizados por Trabajadores/as sociales y llevados a cabo en el ámbito educativo.

4. Mediación: Un/a trabajador/a social especializado en mediación familiar puede ser una buena opción de cara a la gestión de conflictos, canalización del estrés... 


\section{CONCLUSIONES}

Siguiendo los resultados de estudios, los/as adolescentes pueden llegar a pasar demasiado tiempo utilizando las TIC en cualquiera de sus formas durante su día a día pudiendo ser relativamente sencillo que los/as mismos/as lleguen a desarrollar una adicción a todo lo que engloban y ofrecen dichas TIC

Según investigaciones realizadas, la familia es uno de los sistemas sino el sistema más afectado por la aparición de una adicción a las TIC en adolescentes, ya que una situación de este tipo puede llevar a la pérdida de aspectos tan elementales en una familia como la dinámica familiar, la comunicación, el respeto, etc.

La irrupción de las TIC ha supuesto un verdadero dilema tanto para los/as jóvenes que acaban desarrollando una adicción hacia ellas, como para numerosas familias que se debaten continuamente entre el no poder mantener a sus hijos/as alejados/as de las mismas, debido a que la aplastante realidad indica que hoy en día la tecnología prima, y el miedo a que éstos/as acaben desarrollando un círculo vicioso y nocivo en relación a su uso.

Siguiendo la última encuesta realizada por el INE (2018), a la edad 15 años, prácticamente todos/as las/os adolescentes manejan y poseen dispositivos digitales, siendo un poco más acusado el porcentaje cuando hablamos de chicas.

La última versión del manual diagnóstico DMS-V deja una puerta abierta para la inclusión en su próxima actualización del estudio de la adicción por parte de los jóvenes a juegos de internet debido a la incidencia del tema

La OMS prepara la inclusión en la Clasificación Internacional de Enfermedades (no actualizada desde 1990) el trastorno por videojuegos en jóvenes como problema mental

En 2018, la adicción a las TIC ha entrado por primera vez en el Plan Nacional de Adicciones y señala a los/as adolescentes como el grupo de mayor riesgo debido a la creciente incidencia de la problemática en este sector de población.

Es importante trabajar el problema de adicción a las TIC en adolescentes con los progenitores de los/as mismos/as pues es fundamental la adquisición de habilidades que permitan hacer frente de la mejor forma posible a este tipo de situaciones que se pueden llegar a vivir

El Trabajo Social por su carácter asistencial, su visión holística de intervención y su extensa experiencia histórica en problemáticas relacionadas con juventud, familia y adicciones, se configura como una disciplina idónea y necesaria en el tratamiento de adicciones a las TIC.

\section{BIBLIOGRAFÍA}

Álvarez, O. J. \& Rodríguez, E. (2012). El uso de Internet y su influencia en la comunicación familiar. Trilogía (7), 81101. Recuperado de: https://dialnet.unirioja.es/servlet/articulo? codigo $=4521388$. Acceso 02/06/19

Ángel, M. B. \& Alzate, Y. E. (2015). Relaciones familiares y sociales en adolescentes usuarios de redes sociales virtuales (RSV). Katharsis, (20). Recuperado de: https://dialnet.unirioja.es/servlet/articulo? codigo $=5585568$. Acceso 13/06/2019

Asociación Americana de Psiquiatría (2016). Manual diagnóstico y estadístico de trastornos mentales ( $5^{\mathrm{a}}$ ed. Texto rev.). Washington, DC, EE.UU. Recuperado de: https:// dsm.psychiatryonline.org/pb-assets/dsm/update/Spanish DSM5Update2016.pdf. Acceso 28/10/2019

Atienza, J. (2017). Mala educación más que adicción a las TIC. Madrid, España: MásQueNegocio. Movistar. Recuperado de: https://www.masquenegocio.com/2017/05/11/ adiccion-ticl. Acceso 25/06/2019

Castro, C. \& Rodríguez, E. (2016). Intervención social con adolescentes: Necesidades y recursos. Trabajo Social Hoy, (77), 7-23. Recuperado de: https://dialnet.unirioja.es/servlet/articulo?codigo $=5618171$. Acceso 21/06/2019

Centro de Investigaciones Sociológicas. (Marzo, 2016). Barómetro de Marzo 2016: Avance de resultados. Recuperado de: http://www.rtve.es/contenidos/documentos/barometro cis marzo 2016.pdf.

Domínguez, A. M. (22 de Septiembre, 2017). ¿Yo adicto? Diario Cubano Juventud Rebelde. Recuperado de: http:// www.juventudrebelde.cu/cuba/2017-06-20/yo-adicto. Acceso 20/06/2019

Echeburúa, E. y De Corral, P. (2010). Adicción a las nuevas tecnologías y a las redes sociales en jóvenes: un nuevo reto. Adiciones, 22 (2), 91-95. Recuperado de: https:// www.researchgate.net/publication/44449242 Adiccion a las nuevas tecnologias y a las redes sociales en jovenes un nuevo reto. Acceso 25/06/2019

García, C. Piqueras, J. A. y Marzo, J. C. (2017). Uso problemático de Internet, el móvil y los videojuegos en una muestra de adolescentes alicantinos. Health and addictions. Salud y drogas, 17 (2), 189-200. Recuperado de: https://ojs.haaj.org/index.php/haaj/article/view/331/pdf. Acceso 07/10/2019

Garrote, G. (2013). Uso y abuso de tecnologías en adolescentes y su relación con algunas variables de personalidad, estilos de crianza, consumo de alcohol y autopercepción como estudiante. (Tesis inédita). Universidad de Burgos, 
Facultad de Humanidades y Educación, España. Recuperado de: https://riubu.ubu.es/bitstream/handle/10259/219/ Garrote P\%E9rez de Alb\%E9niz.pdf;jsessionid=ADCFD68804BBA174067C275AEA2CB173? sequence $=1$. ACceso 25/06/2019

Instituto Nacional de Estadística. (7 de Noviembre, 2018). Encuesta sobre el Equipamiento y Uso de Tecnologías de Información y Comunicación en los Hogares. Recuperado de: https://www.ine.es/prensa/tich 2018.pdf. Acceso 07/07/2019

Los tres criterios para saber si eres adicto a los videojuegos, según la OMS. (2018, 3 de Enero). BBC Mundo. Recuperado de: http://www.bbc.com/mundo/noticias-42554655. Acceso 07/07/2019

Luengo, A. (2004). Adicción a Internet: conceptualización y propuesta de intervención. Revista Profesional Española de Terapia Cognitivo-Conductual, 2 (1), 22-51. Recuperado de: http://www.jogoremoto.pt/docs/extra/BL5L6u.pdf. Acceso 03/06/2019

Muñoz, N. E. \& Ramírez, S. (2016). Adicción a las redes sociales y personalidad, en adolescentes. Psicoeducativa: reflexiones y propuestas, 2 (4), 46-52. Recuperado de: http://docplayer.es/47568447-Adiccion-a-las-redes-sociales-y-personalidad-en-adolescentes.html. Acceso 14/07/2019

Pedrero, E. J, Rodríguez, M. T. \& Ruiz, J. M. (2012). Adicción o abuso del teléfono móvil. Revisión de la literatura.
Adicciones, 24 (2), 139-152. Recuperado de: http://www. adicciones.es/index.php/adicciones/article/view/107. Acceso 20/07/2019

Salas, J. (21 de Diciembre de 2017). La OMS reconoce el trastorno por videojuegos como problema mental. El País. Recuperado de: https://elpais.com/elpais/2017/12/21/ciencia/1513852127 232573.htm Acceso 07/07/2019

Segura, C. (11 de Marzo de 2018). España se prepara para una nueva adicción. El País. Recuperado de: https://elpais.com/ politica/2018/03/02/actualidad/1520012219 058442. html. Acceso 16/08/2019

Sigüenza, R. F. \& Fernández, G. V. (2016). Nivel de adicción al Internet y comportamiento adictivo de los niños de sexto y séptimo grado de la escuela Carlos Crespi. Cuenca 2016. (Trabajo fin de Grado inédito). Universidad de Cuenca, Facultad de Ciencias Médicas, Ecuador. Recuperado de: http://dspace.ucuenca.edu.ec/bitstream/123456789/26956/1/PROYECTO\%20DE\%20INVESTIGACI\%C3\%93N.pdf. Acceso: $16 / 06 / 2019$

Téllez, J. C. (3 de Marzo de 2018). La "adicción" a las nuevas tecnologías enfrenta a la OMS con el sector de los videojuegos. Vozpópuli. Recuperado de: https://www.vozpopuli.com/altavoz/tecnologia/adiccion-tecnologia-OMS-videojuegos-trastorno-adicto-droga 0 1113789249.html. Acceso 07/08/2019 